\title{
Discrimination learning in humans: Role of number and complexity of rules
}

\author{
J. H. R. MAes AND P. A. T. M. Eling \\ Radboud University Nijmegen, Nijmegen, The Netherlands
}

\begin{abstract}
Various types of discrimination learning tasks, such as so-called nonconditional, conditional, and biconditional tasks, are generally held to differ in complexity and to require different amounts of training. However, rather than a difference in rule complexity, between-task performance differences may reflect a difference in number of underlying rules. Accordingly, in the present study, human participants were subjected to tasks differing in number and/or complexity of rules. In Experiments 1 and 3, participants learned to differentially respond to visual-target stimuli, each of which was preceded by a visual feature. Conditions differed in the number of different features and in the informational value of individual features and/or targets. In Experiment 2, participants were fully informed about all relevant stimulus-response mappings prior to each trial. Performance accuracy was primarily determined by number of underlying rules in the initial phase of discrimination learning, especially when the time available for responding was restricted. However, when participants had attained a high accuracy level, performance was solely determined by rule complexity. Apparently, number and complexity of rules have a different weight, depending on the stage of discrimination learning.
\end{abstract}

All discrimination learning, that is, learning to respond differentially to distinct target stimuli, can be considered to be "conditional learning:" Experimental subjects have to learn that given a particular stimulus, only a specific response is correct. However, in the literature on human and animal discrimination learning, a distinction is made between conditional and nonconditional tasks. Tasks are termed nonconditional if correct responding to a target stimulus is not conditional upon the presence or absence of some other, nontarget, stimulus. An example is a task requiring a left-key response (R1) to Stimulus 1 (T1) and a right-key response (R2) to Stimulus 2 (T2). In a conditional discrimination task, correct responding to a target does depend on the presence or absence of a preceding or cooccurring stimulus, hereafter termed a feature. For example, T1 requires R1 if accompanied by one feature (F1), but it requires R2 if accompanied by another feature (F2) (schematically, F1: T1 $\rightarrow$ R1, F2: T1 $\rightarrow$ R2). However, importantly, this particular discrimination task also allows for a nonconditional solution: Correct responding is also possible by simply matching each feature with the correct response. In order to preclude such a solution, one needs an experimental design incorporating at least two features and two targets: F1 indicates that R1 is the correct response to $\mathrm{T} 1$ and that $\mathrm{R} 2$ is the correct response to $\mathrm{T} 2$. Moreover, F2 signals that R2 is the correct response to T1 and that R1 is the correct response to T2 (schematically, $\mathrm{F} 1: \mathrm{T} 1 \rightarrow \mathrm{R} 1, \mathrm{~F} 1: \mathrm{T} 2 \rightarrow \mathrm{R} 2, \mathrm{~F} 2: \mathrm{T} 1 \rightarrow \mathrm{R} 2, \mathrm{~F} 2: \mathrm{T} 2 \rightarrow \mathrm{R} 1$; see Table 1, Condition F2+T2). In this case, often referred to as a biconditional discrimination, neither feature nor target alone is sufficient to determine the correct response; the task requires learning about feature/target conjunctions. However, even in this case, one cannot be sure whether an experimental subject will solve the task in such a "conditional" manner. For example, an alternative approach might be that each feature/target combination is treated as a unique cue that is associated with a particular response.

Given these ambiguities with respect to the notion of conditionality, and an uncertainty as to how a particular subject might interpret a particular stimulus sequence, perhaps a better description of the different types of tasks can be based on two theoretical frameworks that can be discerned in the literature. One is primarily linked to human experimental work on concept or rule learning; the other to animal studies on discrimination learning.

In a typical human concept or rule-learning experiment, participants have to acquire information on the assignment of combinations of values of relevant stimulus dimensions to response categories. Most of the corresponding studies employed stimuli consisting of two or more dimensions, such as colored geometrical figures, implying the simultaneous presence of multiple dimensions. However, one could also apply this framework to learning tasks involving a serial presentation of different stimuli. Thus, applied to the example of a biconditional task, a serially presented feature and target stimulus may be represented as two "dimensions," each with two possible "values," and the participant has to learn to assign different stimulus combinations to either R1 or R2. In the 1960 s, concept learning was a topic receiving considerable experimental

J. H. R. Maes, r.maes@nici.ru.nl 
Table 1

Outline of Trial Types and Characteristics for Each Condition

\begin{tabular}{|c|c|c|c|}
\hline Condition & Trial Types & No. of Rules & $\begin{array}{c}\text { Rule } \\
\text { Complexity }\end{array}$ \\
\hline $\mathrm{F} 2$ & $\begin{array}{l}\mathrm{F} 1: \mathrm{T} 1 \rightarrow \mathrm{R} 1 \\
\mathrm{~F} 1: \mathrm{T} 2 \rightarrow \mathrm{R} 1 \\
\mathrm{~F} 2: \mathrm{T} 1 \rightarrow \mathrm{R} 2 \\
\mathrm{~F} 2: \mathrm{T} 2 \rightarrow \mathrm{R} 2\end{array}$ & $\begin{array}{l}\text { (1) if F1: R1 } \\
\text { (2) if F2: R2 }\end{array}$ & 1 \\
\hline $\mathrm{T} 2$ & $\begin{array}{l}\mathrm{F} 1: \mathrm{T} 1 \rightarrow \mathrm{R} 1 \\
\mathrm{~F} 1: \mathrm{T} 2 \rightarrow \mathrm{R} 2 \\
\mathrm{~F} 2: \mathrm{T} 1 \rightarrow \mathrm{R} 1 \\
\mathrm{~F} 2: \mathrm{T} 2 \rightarrow \mathrm{R} 2\end{array}$ & $\begin{array}{l}\text { (1) if T1: R1 } \\
\text { (2) if T2: R2 }\end{array}$ & 1 \\
\hline F4 & $\begin{array}{l}\mathrm{F} 1: \mathrm{T} 1 \rightarrow \mathrm{R} 1 \\
\mathrm{~F} 2: \mathrm{T} 1 \rightarrow \mathrm{R} 2 \\
\text { F3: T2 } \rightarrow \mathrm{R} 1 \\
\text { F4: T2 } \rightarrow \mathrm{R} 2\end{array}$ & $\begin{array}{l}\text { (1) if F1: R1 } \\
\text { (2) if F3: R1 } \\
\text { (3) if F2: R2 } \\
\text { (4) if F4: R2 }\end{array}$ & 1 \\
\hline $\mathrm{T} 4$ & $\begin{array}{l}\mathrm{F} 1: \mathrm{T} 1 \rightarrow \mathrm{R} 1 \\
\mathrm{~F} 1: \mathrm{T} 2 \rightarrow \mathrm{R} 2 \\
\mathrm{~F} 2: \mathrm{T} 3 \rightarrow \mathrm{R} 1 \\
\text { F2: T4 } \rightarrow \mathrm{R} 2\end{array}$ & $\begin{array}{l}\text { (1) if T1: R1 } \\
\text { (2) if T3: R1 } \\
\text { (3) if T2: R2 } \\
\text { (4) if T4: R2 }\end{array}$ & 1 \\
\hline $\mathrm{F} 2+\mathrm{T} 2$ & $\begin{array}{l}\mathrm{F} 1: \mathrm{T} 1 \rightarrow \mathrm{R} 1 \\
\mathrm{~F} 1: \mathrm{T} 2 \rightarrow \mathrm{R} 2 \\
\mathrm{~F} 2: \mathrm{T} 1 \rightarrow \mathrm{R} 2 \\
\mathrm{~F} 2: \mathrm{T} 2 \rightarrow \mathrm{R} 1\end{array}$ & $\begin{array}{l}\text { (1) if } \mathrm{F} 1+\mathrm{T} 1: \mathrm{R} 1 \\
\text { (2) if } \mathrm{F} 2+\mathrm{T} 2: \mathrm{R} 1 \\
\text { (3) if } \mathrm{F} 1+\mathrm{T} 2: \mathrm{R} 2 \\
\text { (4) if } \mathrm{F} 2+\mathrm{T} 1: \mathrm{R} 2\end{array}$ & 2 \\
\hline
\end{tabular}

Note-F1, F2, F3, and F4 refer to different features, and T1, T2, T3, and $\mathrm{T} 4$ are targets used to create the different trial types. $\mathrm{R} 1$ and $\mathrm{R} 2$ represent different required responses. The number of rules is based on the number of simplest possible propositional statements necessary to define the correct response. Rule complexity refers to the maximum number of elements (features and/or targets) within each such rule.

and theoretical attention (see, e.g., Haygood \& Bourne, 1965 , for a review), and a resurgence of interest can be noted in more recent articles (e.g., Feldman, 2003). In this literature, conceptual rules are classified in terms of complexity levels (see, e.g., Halford, Wilson, \& Phillips, 1998; Haygood \& Bourne, 1965), sometimes using a mathematical measure, such as the so-called Boolean complexity (e.g., Feldman, 2000, 2006). The simplest rule, the "affirmation" rule (see, e.g., Bourne, 1967), defines one value of a specific relevant binary dimension as belonging to a specific response class. For example, let us consider an effectively nonconditional variant of the biconditional task indicated above: F1: T1 $\rightarrow \mathrm{R} 1, \mathrm{~F} 1: \mathrm{T} 2 \rightarrow \mathrm{R} 2, \mathrm{~F} 2: \mathrm{T} 1 \rightarrow \mathrm{R} 1$, and F2:T2 $\rightarrow$ R2 (see Table 1: Condition T2). This task implies the same number of features, targets, and responses as does the biconditional task, but there is, in fact, only one rule that defines belongingness to the R1 category, namely "if $\mathrm{T} 1$," and one rule defining belongingness to the alternative, R2 category, "if T2." In the biconditional task, a rule defining the $\mathrm{R} 1$ response category is more complex than is the case for the task in Condition T2: It entails the conjunction of one value of each of the two dimensions-for example, "if F1 and T1." A further difference is that two such rules are required to exhaustively define R1 category membership (namely also "if F2 and T2") instead of only one. A robust empirical finding is that the difficulty of the task - as, for example, measured by learning rate - is inversely related to the complexity of the task, as expressed by some more or less formal complexity measure (see, e.g., Bourne, 1967).

In the 1980s and 1990s, numerous animal conditioning experiments explored the nature of the associations that are established in Pavlovian conditional discrimination tasks in which features and targets are typically presented serially instead of simultaneously (see, e.g., Swartzentruber, 1995). Specifically, the features signal whether or not a specific target stimulus will be followed by a biologically significant stimulus (unconditioned stimulus, or US). A common finding is that animals like rats and pigeons are able to solve these kinds of tasks: The animals come to almost exclusively respond to the target stimulus if it is preceded by a feature that explicitly signals that the target will be followed by the US. Importantly, this behavior does not seem to rest upon the establishment of a simple association between a feature or target and the US (or the absence thereof). Instead, the features seem to function in a more complex, hierarchical way, as "occasion setters" for a specific target-US relationship. As indicated before, an alternative approach in this literature is to explain appropriate responding in (bi) conditional tasks in terms of the subjects treating each feature-target combination as a configural or unique stimulus (e.g., Pearce, 1994; Rescorla, 1973) that may or may not be associated with a US. However, such a solution seems to be especially encouraged in the case of a simultaneous presentation of features and targets.

To the authors' knowledge, a direct comparison of the learning rate in rats that were subjected to biconditional and "nonbiconditional" discrimination tasks was only made in one previous study (Maes \& Vossen, 2001). Much slower learning was observed in a biconditional task than in a task that was conceptually identical to Condition T2 in Table 1. This large performance difference is comparable to the difference in learning speed observed in human concept learning tasks that differ in complexity of underlying rules.

In previously published human concept-learning studies, rule complexity seems to be implicated as the most important determinant of learning rate. However, a confounding factor in at least a number of reported comparisons might be a difference in the mere number of underlying rules, regardless of their complexity. For example, a biconditional task - as in Condition F2 + T2 (Table 1) implies four rules, whereas each of the conditions that involve the same number of features and targets, but that fail to have a biconditional structure - Conditions F2 and T2 (Table 1) -imply only two rules. Assuming an even distribution of the four different trial types, this means that the opportunity to test the relevant rules in each of the latter tasks is twice as great as that in the biconditional task.

Using a conceptual framework from the animal literature, another way of expressing this notion is that within each block of four different trial types, the association between the relevant stimulus and the appropriate response can grow twice as fast in tasks implying two rules as in a task implying four rules. This is simply because the number of to-be-learned S-R associations is greater in the former tasks than in the latter task. For example, in a set of four trials in Condition F2, the $\mathrm{F} 1 \rightarrow \mathrm{R} 1$ association can increase on two trials, whereas in Condition F2+T2, each feature-target combination is associated with the appropriate response only once.

The purpose of the present series of experiments was to test the hypothesis that differences in learning rate for 
humans in tasks that are conceptually similar to animal discrimination-learning tasks can be explained in terms of number of underlying rules.

\section{EXPERIMENT 1}

Four experimental conditions were included in Experiment 1 . In each condition, the participants had to learn which one of two possible responses (R1 or R2) had to be performed after the presentation of a target stimulus. The conditions used in Experiment 1 (see Table 1: all conditions except for Condition T4) differed in the number and/or informational value of targets and features. Specifically, for the participants in Condition F2, the two target stimuli (T1 and T2) were not informative with respect to the correct response. However, correct responding was possible on the basis of two features (F1 and F2). Participants in Condition T2 could base their response on two targets, but not on the two features. Conditions F2 and T2 contained the same number of features and targets as that in Condition F2 + T2. However, in the latter condition, neither features nor targets alone, but only feature-target combinations, enabled choice of the correct response. One additional condition, Condition F4, was included in which the number of to-belearned rules was increased in comparison with the F2 and $\mathrm{T} 2$ conditions. This was achieved by increasing the number of features by two. In this condition, correct responding was possible on the basis of the features alone. As can be seen in Table 1, the conditions differed with respect to number of required rules and/or complexity of the rules.

If the number of underlying rules is the only factor of importance, the expected learning-rate order is: $\mathrm{F} 2=\mathrm{T} 2>$ $\mathrm{F} 4=\mathrm{F} 2+\mathrm{T} 2$. However, if difficulty is determined by both the number of underlying rules and rule complexity, the expected order would be $\mathrm{F} 2=\mathrm{T} 2>\mathrm{F} 4>\mathrm{F} 2+\mathrm{T} 2$.

\section{Method}

Participants. Forty students, 8 male and 32 female, ranging in age from 18 to 26 years, volunteered to participate. The students were randomly assigned to one of four experimental conditions $(n=$ 10 ), each containing 1-3 males. The participants were tested in their home environment.

Apparatus. The experiment was run on a laptop with a 15-in. screen using the experiment-generator program, E-prime. The visual stimuli used in the experiment were presented against a white background and consisted of a circle (diameter: $5 \mathrm{~cm}$ ) that was either red, blue, orange, or purple, and a rectangle $(5 \mathrm{~cm} \times 2.5 \mathrm{~cm})$ that was colored green or yellow. Responses that were recorded consisted of pressing either the letter "Z" ("left response;" R1) or "M" ("right response;" R2). Response feedback consisted of either the word "Correct," written in blue, or the word "Incorrect," written in red, with both words presented against a white background.

Procedure. The participant was seated in front of the computer screen and read the following instructions:

Welcome to the experiment. You will see a figure followed by another figure. It is your task to give a response as soon as you see the second figure. Choose between pressing the " $Z$ " key and the "M" key. You will receive information about whether your answer was correct or incorrect. Work as fast as you can and try to give as many correct answers as possible.

A trial consisted of the presentation of one of the circles, which was used as a feature, for $800 \mathrm{msec}$, followed by a blank screen for
$1,000 \mathrm{msec}$. Subsequently, one of the rectangles serving as a target, was presented with a maximum duration of 1,200 msec. Feedback about response correctness was given if the participant made a response to the target within this $1,200 \mathrm{msec}$. Feedback was presented for $1,500 \mathrm{msec}$. If a subject did not respond within 1,200 msec after target-stimulus onset, the words "too late" were presented as feedback, and the current trial was immediately repeated until a "Z" or " $M$ " response was made. In this way, 80 trials with a valid response were collected for each participant.

Each participant was presented with four different trial types. The nature of the trial types depended on the experimental condition that the participant was assigned to, as shown in Table 1 (all conditions except Condition T4). In this table, F1, F2, F3, and F4 represent a red, blue, orange, and purple circle, respectively. T1 and T2 signify, respectively, a green and yellow bar, and R1 and R2 designate a left- and right-key press, respectively. Trial types were presented semirandomly, with each trial type occurring once in each block of four trials.

Dependent measure. The main dependent measure in this experiment, as well as in Experiments 2 and 3, was the percentage of correct responses across trial blocks, which was subjected to a condition $\times$ trial block repeated measures ANOVA. Scheffé's post hoc tests were used to further evaluate differences between conditions. On reporting the results of these latter tests, subsets of conditions with nonsignificant differences are indicated. The level of significance was set at $p<.05$ for all statistical analyses.

\section{Results and Discussion}

The overall mean number of trials with no response to the target within the 1,200-msec time limit (thereby causing the trial to be repeated) was 4.1. An ANOVA on the number of trials with no response did not reveal a significant difference among the conditions $[F(3,40)<1]$.

Figure 1 shows for each condition the percentage of correct responses across blocks of 20 trials. Note that the participants in each of the Conditions F2 + T2 and F4 were slower in learning the task than were those in each of the Conditions F2 and T2.

An ANOVA on the data depicted in Figure 1 revealed significant main effects of condition $[F(3,36)=15.34$, $p<.001]$, and trial block $[F(3,108)=16.24, p<.001]$. The condition $\times$ trial block interaction was not significant $[F(9,108)<1, p=.81]$. Post hoc analysis revealed two homogeneous subsets: Conditions F2 and T2 $(p=.930)$, and Conditions F4 and F2+T2 $(p=.723)$.

These results suggest that learning rate was mainly affected by the number of rules and that rule complexity was relatively unimportant. However, performance accuracy reached by the participants in Conditions F4 and F2+T2 still was quite low at the end of the session. Therefore, the question remains whether rule complexity is the dominant factor if participants have mastered the task - that is, when learning processes no longer play an important role. Hence, in Experiment 2, we explicitly instructed the participants about the stimulus-response mappings prior to performing the task.

\section{EXPERIMENT 2}

Previous research on rule learning suggests that rule induction or learning is a process that is fundamentally different from rule application and that these processes are subserved by different neuronal structures. For example, 


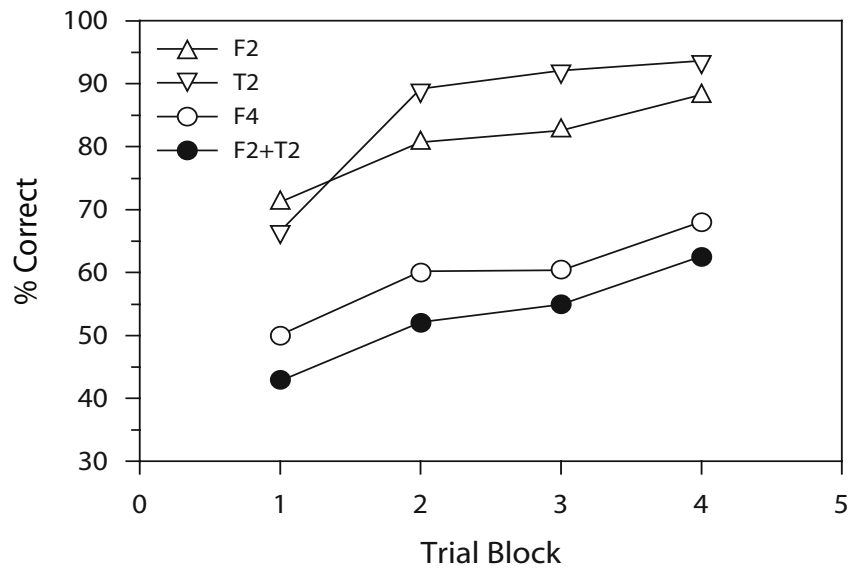

Figure 1. Mean percentage correct responses for each condition across blocks of 20 trials in Experiment 1. The conditions differed in the number and/or informational value of feature and target stimuli. The task in Conditions F2, T2, and F4 could be solved by learning simple stimulus-response mappings. Condition $\mathrm{F} 2+\mathrm{T} 2$ required the learning of responses to feature/target conjunctions.

researchers have suggested that the dorsolateral prefrontal cortex is specifically implicated in "higher order" rule learning, whereas the fronto-polar cortex and the superior frontal gyrus are explicitly involved in the use of such rules (see, e.g., Boettinger \& D'Esposito, 2005). Therefore, Experiment 2 asked whether the observed predominance of the number of required rules - rather than of the complexity of rules - revealed in Experiment 1 would also hold in a rule-execution task in which the performance rules were fully known to the participants in advance. The experimental conditions in Experiment 2 were the same as those in Experiment 1. However, instead of the participants having to infer the correct rule on a trial-by-trial basis, a summary of the underlying rules was presented prior to each trial.

\section{Method}

Participants and Apparatus. Volunteers were 18 male and 22 female students (age range: 18-29 years). Participants were assigned to one of four experimental conditions $(n=10)$. The sex ratio was roughly equivalent in the conditions. Stimuli and apparatus were the same as those in Experiment 1.

Procedure. The instructions for the participants in Condition F2 were identical to those used in Experiment 1 for the corresponding condition except for the inclusion of the following sentences:

Press " $Z$ " when the first figure was a blue circle. Press " $M$ " when the first figure was a red circle. This instruction will be presented just before each trial for as long as you wish. You will not receive any feedback if you respond according to the instructions.

Prior to each trial, a summary of the rule ("Blue circle $=\mathrm{Z}$, Red circle $=$ M") was presented until the participant pressed the space bar. The trial started immediately after a space-bar response. The words "too early" were presented as feedback for $1 \mathrm{sec}$ if the participant responded (pressing either one of the two response options "Z" and " $M$ ") during the feature presentation or during the featuretermination and target-onset interval. The words "too late" were presented as feedback for $1 \mathrm{sec}$ if no valid response (either correct or incorrect) was made during the 1,200-msec target stimulus presentation. Finally, the word "Wrong!" was presented for $1 \mathrm{sec}$ upon mak- ing an incorrect response. Unlike in Experiment 1, the just-presented trial was repeated following a response that either was too early, too late, or incorrect, thereby ensuring the collection of 80 trials with a correct response for each subject. The collection of 80 correct trials was important in view of our original intention to analyze and report response times (RTs) in addition to performance accuracy. However, in view of the present research question, the RT analyses did not yield any useful additional information. Therefore, and for the sake of conciseness, RT data will not be reported in this article.

The instruction and rule summary were correspondingly adapted for each of the other conditions. For example, the rule summary for Condition T2 was "Green bar = Z, Yellow bar = M," and for Condition F2 + T2 it was "Red circle + Green bar $=Z$, Blue circle + Yellow bar $=$ Z, Blue circle + Green bar $=M$, Red circle + Yellow bar = M." Importantly, the participants were encouraged to study the instructions very carefully and to try and derive a rule that would be most easy for them to memorize. All further details for these conditions were as those described for Condition F2.

\section{Results and Discussion}

The mean total number of trials needed to attain 80 trials with a correct response was 85.3 for Condition F2, 81.6 for Condition T2, 87.1 for Condition F4, and 95.4 for Condition F2+T2. An ANOVA with condition as the betweensubjects factor revealed a significant effect $[F(3,36)=$ $8.50, p<.001]$, which reflected the fact that Conditions F2, T2, and F4 formed one homogeneous subset (Scheffé, $p=.30$ ), and Condition F2+T2 another one.

Figure 2 depicts the mean percentage correct responses across 20 -trial blocks on the basis of the first 80 trials. Conditions F2 and T2 showed a near-asymptotic response accuracy from Block 1 on. The participants in Condition F4 clearly showed a learning effect from Block 1 to Block 2 and Condition F2+T2 displayed response accuracy that clearly was enhanced in comparison with that shown at the end of training in Experiment 1.

However, of primary importance, across trial blocks, the performance in Condition F2 + T2 remained at a lower level than in each of the other three conditions. An ANOVA on the data shown in Figure 2 revealed a main effect of trial block $[F(3,108)=4.40, p<.01]$, but no condition $\times$ trial block interaction $[F(9,108)=1.42, p=.18]$. The main effect of condition was significant $[F(3,36)=12.10$, $p<.001]$. There were two homogeneous subsets: Conditions F1, T2, and F4 ( $p=.82)$, and Condition F2+T2.

These results indicate that under the condition of explicitly instructing the stimulus-response mappings prior to each trial, yielding a (relatively) high response accuracy in all conditions, rule complexity was the critical factor in determining performance. Figure 2 clearly suggests learning effects in all conditions, also illustrating the difficulty in separating "learning" from "performance." However, the hypothesis that emerges from the results of Experiments 1 and 2 is that the weight of the number-of-rules factor is larger than the rule-complexity factor when rule induction is more dominant than rule application and that the weight of these factors reverses if learning processes are (nearly) complete - that is, when response accuracy has reached a relatively high level (larger role for rule application). In Experiment 3, this hypothesis was tested more explicitly by gathering, in a single session, a more elaborate learning function from the participants in each condition. 


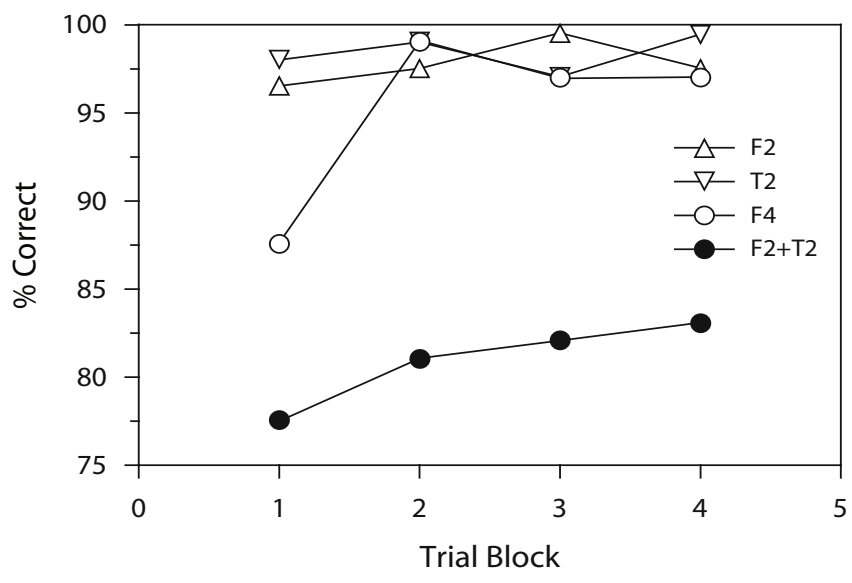

Figure 2. Mean percentage correct responses for each condition across 20 trial blocks in Experiment 2. Participants were instructed about the stimulus-response mappings prior to each trial. The conditions differed with respect to the number and/ or informational value of feature and target stimuli, as shown in Table 1.

\section{EXPERIMENT 3}

In our final experiment, we attempted to assess the effects of the rule number and rule complexity using an experimental design that was expected to yield better task acquisition and, hence, more complete learning than that obtained in Experiment 1. Moreover, a further experimental condition was included that implied a rule complexity of " 1 " and the use of four rules, namely T4 (see Table 1). A number of features were introduced that were thought to encourage better discrimination performance in all conditions. First, the number of training trials was increased from 80 to 208 . Second, there was no time limit for giving a response to the target. Third, the difference between features and targets was made more salient than was the case in Experiment 1. Fourth, feedback about response accuracy was given immediately after each trial, presumably (additionally) motivating the participants to increase response accuracy.

If these modifications resulted in better discrimination performance, then on the basis of the results of Experiments 1 and 2, we expected that participants in Conditions F4, T4, and F2 + T2 (each implying four rules) would perform worse than would the participants in Conditions F2 and T2 (two rules) in the first phase of discrimination learning (i.e., at least across the first 80 training trials; see Figure 1). However, after reaching a minimum level of performance accuracy, as occurred in Experiment 2, response accuracy would mainly be determined by rule complexity, implying lower performance accuracy in Condition F2 + T2 than in each of the other four conditions.

\section{Method}

The participants were 23 males and 27 females ranging in age from 20-24 years. Each student was assigned to one of five conditions. The number of males and females was about equal in each condition. Four conditions were as those described in the previous experiments (F2, T2, F4, and F2 + T2); one additional condition was
T4 (see Table 1). There were four different target stimuli in this latter condition. Two of these required R1 as the correct response, whereas the other two required R2 as the correct response. All further details were like those in Experiment 1, except for the following. The features F1, F2, F3, and F4 consisted of a gray circle, triangle, star, and square. The targets consisted of a green, blue, red, or yellow bar. The number of trials was 208 , and the target stimulus remained visible until the participant either made a "Z" or "M" response. Feedback about response accuracy was provided immediately after a response. Following a correct response, the words "well done" appeared, together with information regarding the percentage of trials on which the participant had given a correct response. If the participant made an incorrect response, the word "incorrect" was presented. The order of trial types was random.

\section{Results and Discussion}

Figure 3 displays the percentage correct responses across blocks of 26 trials. An ANOVA on the corresponding data revealed a significant main effect of trial block $[F(7,315)=37.60, p<.001]$ and condition $[F(4,45)=$ $9.24, p<.001]$. The interaction effect was not significant $[F(28,315)<1]$. A post hoc analysis revealed that Conditions F2, T2, F4, and T4 belonged to one subset ( $p=.64)$, and Condition $\mathrm{F} 2+\mathrm{T} 2$ to another.

To compare the present data with those from Experiment 1 , a separate condition $\times$ trial Block ANOVA was performed using the data from the first three trial blocks in order to examine differences between conditions in the initial phase of the learning process. These blocks correspond to the first 78 trials, which very nearly correspond to the 80 trials presented in Experiment 1 (see Figure 1). The ANOVA revealed a main effect for trial block $[F(2,90)=$ $26.95, p<.001]$ and condition $[F(4,45)=5.70, p=$ $.001]$, and no interaction effect $[F(8,90)<1]$. A post hoc test revealed two subsets: Conditions F4, T4, and F2+T2 $(p=.06)$, and Conditions F2, T2, F4, and T4 ( $p=.68)$.

These results suggest that - albeit not as clearly as was the case in Experiment 1-the number of underlying rules is a factor of importance in the initial phase of

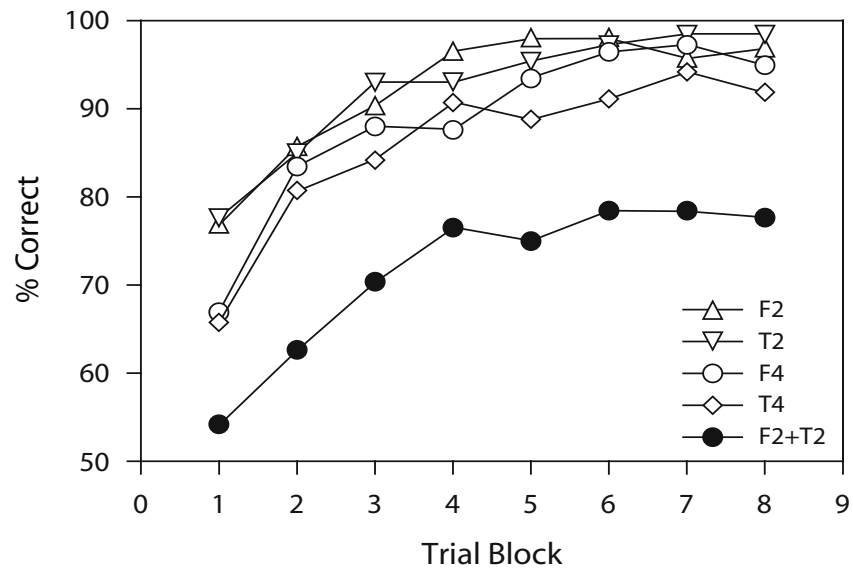

Figure 3. Mean percentage correct responses across blocks of 26 trials in Experiment 3. Participants had to induce the correct response to the features and/or targets on the basis of feedback, as they did in Experiment 1. See Table 1 for an explanation of the trial types used in each condition. 
discrimination learning: All conditions that implied four underlying rules, F4, T4, and F2 + T2, formed one homogeneous subset. However, after obtaining a certain level of performance accuracy, rule complexity was the dominant factor, as indicated by the fact that all conditions with rule complexity 1 (F2, T2, F4, and T4) formed one homogeneous subset, and that the condition with rule complexity $2(\mathrm{~F} 2+\mathrm{T} 2)$ belonged to a separate subset.

\section{GENERAL DISCUSSION}

The present experiments revealed that the performance in a biconditional task involving two features, two targets, and two responses (Condition F2+T2) was worse than that in two versions involving the same number of features, targets, and responses, but lacking a biconditional structure (Conditions F2 and T2). This finding is consistent with the results of a previous animal study (Maes \& Vossen, 2001). In that study, rats trained on a task that was conceptually identical to the present Condition F2+T2 displayed a much slower learning rate than did rats in a task that was conceptually identical to the present Condition T2.

Comparisons with two additional tasks that also lacked a biconditional structure, but that involved a larger number of features or targets (Conditions F4 and T4), allowed us to further examine possible reasons for the impaired performance in Condition F2+T2. These comparisons revealed that the learning rate was solely (Experiment 1) or importantly (Experiment 3 ) determined by the number of basic rules required for adequate responding, as reflected in similar learning in Conditions F4, T4, and $\mathrm{F} 2+\mathrm{T} 4$. However, after reaching a performance accuracy of about $>70 \%$ (Experiments 2 and 3), performance seemed to be primarily determined by rule complexity, as indicated by less accurate performance in Condition $\mathrm{F} 2+\mathrm{T} 2$ than in each of the other tasks.

A difference was found in the role played by the number or complexity of task-relevant rules depending on the performance accuracy attained by the participants. When the participants clearly were in the process of rule induction (learning), as evidenced by relatively low accuracy levels, the number of rules related to the number of stimulusresponse mappings was a critical factor in determining task load. However, when the students had reached a high accuracy level, either by explicitly providing the rules or by giving more elaborate training, task execution was solely determined by rule complexity. In other words: The present results suggest that the "special status" of biconditional tasks only becomes apparent in a phase in which rule application rather than rule induction is likely to be the dominant process.

Concerning the number of rules, it is conceivable that participants may eventually have induced even more efficient rules than those described in Table 1. For example, in Condition F4, participants might have come to derive the following rules: (1) If F1 then R1, (2) if F3 then R1, (3) else R2, implying only three instead of four rules. However, the "else" rule can, in principle, also be used in each of the other conditions, implying that Conditions F2 and T2 would still involve fewer rules than Conditions F4, T4, and F2+T2. Similarly, one could argue that the participants in Condition F4, for example, also need only two rules: "if F1 or F3, then R1," and "if F2 or F4, then R2." However, note that such formulation of rules would not be in line with the classification of rule complexity as is commonly used in the literature on rule learning. This classification is made on the basis of the possible combinations of stimulus attributes from two or more stimulus dimensions that define a concept. In each of the conditions F2, T2, F4, and T4, there is only one relevant stimulus "dimension" (either only the feature or the target) implying a similar (lowest) rule complexity: They are all "affirmative" rules in the classification used by Bourne (1967), for example. These conditions only differ with respect to the number of to-be-classified exemplars within this relevant dimension.

The number of rules was less important in Experiment 1 than in Experiment 3. In Experiment 1, the post hoc analysis clearly revealed that the students' performances in Conditions F2 and T2 (two rules) were similar and better than those in Conditions F4 and F2 + T4 (four rules), which did not differ. However, when examining a number of (initial) trials in Experiment 3 that corresponded to the number of trials presented in Experiment 1, apart from a subset consisting of Conditions F4, T4, and F2+ T2 (four rules), post hoc analysis also clearly revealed a subset consisting of the conditions implying rule complexity " 1 " (all conditions except F2 + T4). The procedural differences between Experiments 1 and 3, such as the presence of a time pressure (limited response time available) in Experiment 1 but not in Experiment 3, may have been responsible for the less-marked effect of the number-of-rules factor in Experiment 3 . The procedural differences resulted in a better overall performance in the former than the latter experiment. If the number of rules primarily plays a role when response accuracy is limited, this performance difference encourages a larger difference between the four-rule conditions on the one hand and the two-rule conditions on the other in Experiment 1 than in Experiment 3.

More generally, it is entirely possible that relatively small parametric changes would result in different outcomes in the present experiments. For example, a change in instruction, stimulus characteristics, or temporal variables, such as the interval between features and targets, could have encouraged the participants to approach the tasks in Conditions F2, T2, F4, and T4 in a more conditional way than was assumed in the present study. That is, they might have come to explicitly evaluate the meaning of each target on the basis of the identity of the feature, or vice versa. In that case, the rule complexity for each of these conditions would also be " 2 ," with a corresponding change in outcome of the experiments. Likewise, if, for example, the features and targets would have been presented simultaneously, then the biconditional task might have been solved in a purely configural way in which the participants would have learned to map each of four unique cues to either a left or a right response. This, in turn, would imply rule complexity " 1 " instead of " 2 ." However, the present task parameters with serial presentation and relatively distinct features and targets were explicitly chosen to yield tasks 
that resemble those used in previous (primarily animal) research on occasion setting (see, e.g., Schwartzentruber, 1995). Therefore, it is not unreasonable to assume that the present findings do have some generality with respect to solving this type of learning task.

The influence of the number of rules in Experiments 1 and 3 may be explained in terms of a cognitive, rulelearning account. Accordingly, given an equal distribution of all different trial types, the difference in the number of underlying rules necessarily implies a longer "hypotheses-testing phase" for Conditions F4, T4, and $\mathrm{F} 2+\mathrm{T} 2$ than for Conditions F2 and T2. However, under the condition of (near) complete knowledge of the rules (Experiment 2 and the final trial blocks in Experiment 3), the participants in Condition F2 + T2 had more difficulty performing the task because of a difference in memory load in comparison with each of the other conditions. That is, each rule in Condition F2 + T2 implies the need to remember two pieces of information rather than only one, as is the case in each of the other conditions. Interestingly, the increase in task load that is implied in Conditions F4 and $\mathrm{T} 4$, in comparison with Conditions F2 and T2-in terms of an expansion of the number of exemplars within the relevant dimension (four instead of two items), did not result in a performance difference between Conditions F4 and T4 and Conditions F2 and T2. In other words, under the conditions of Experiments 2 and 3 (later trial blocks), it is rule complexity rather than mere memory load (within the same simple rule structure) that is crucial.

A different conceptual approach to the present findings is that of associative learning. Accordingly, performance in Conditions F2, T2, F4, and T4 reflects the formation of elemental stimulus-response associations or stimulusresponse-outcome (feedback) associations. For example, an F1:T1 $\rightarrow \mathrm{R} 1$ trial in Condition F2 can be conceptualized as a trial during which both stimuli F1 and T1 gain associative strength with R1. Furthermore, a subsequent $\mathrm{F} 2: \mathrm{T} 1 \rightarrow \mathrm{R} 2$ trial can then be considered to be a trial on which both stimuli F2 and T1 are paired with a "no R1" response. Accordingly, both of these stimuli will lose associative strength with $\mathrm{R} 1$ on this trial, implying that the net associative strength of $\mathrm{T} 1$ with $\mathrm{R} 1$ will decrease and that stimulus F2 acquires a negative associative strength with R1. The latter, in turn, implies that F2 is associated with $\mathrm{R} 2$ rather than $\mathrm{R} 1$.

Within this framework, the solution of nonlinear problems, such as the present conditional task, requires the concept of a unique or configural cue (see, e.g., Pearce, 1987, 1994; Rescorla, 1973). Accordingly, an F1:T1 $\rightarrow$ R1 trial is analogous to presenting a unique $\mathrm{F} 1-\mathrm{T} 1$ configuration in addition to the separate stimuli $\mathrm{F} 1$ and $\mathrm{T} 1$. The representation of the unique cue is only activated when the F1-T1 combination is presented; this unique cue may gain and lose associative strength just as the separate stimuli $\mathrm{F} 1$ and $\mathrm{T} 1 \mathrm{can}$. On this view, task solution in Condition $\mathrm{F} 2+\mathrm{T} 2$ rests upon the acquisition of associative strength by the F1-T1, F1-T2, F2-T1, and F2-T2 stimulus configurations with either R1 or "no R1."

It is not unlikely that the associative strength that is acquired by the (elemental and configural) stimuli on the various trial types is positively related to the likelihood of performing either R1 (given a high associative strength) or R2 (given a negative or zero associative strength). Within the framework of computational models of associative learning (see, e.g., Pearce, 1987, 1994; Rescorla \& Wagner, 1972), it is possible to simulate the pattern of results obtained in the present study, at least when incorporating specific assumptions regarding the value of various parameters, such as those representing the salience of the various stimuli. However, the critical point is that similar to what has been said previously for a more cognitive account - at least in the initial phase of the learning process - the difference in the number of different (elemental or configural) stimuli that are presented in the different conditions and that have to be associated with a particular response necessarily results in differences in the number of trials that are required for each stimulus to obtain a certain level of associative strength.

These computational models predict that learning in Condition F2 + T2 will eventually reach asymptotic learning as well (errorless performance), just as was the case for the other tasks in the present experiments. This prediction is consistent with early work on concept learning indicating that participants who are given sufficient training are able to perfectly perform a biconditional task (see, e.g., Bourne, 1967). Interestingly, this interpretation implies no fundamental difference between tasks differing in rule complexity over and above a difference in learning rate.

These different conceptual approaches and the present empirical findings suggest that in studies comparing performance in tasks that differ in rule complexity, such as in so-called biconditional versus (non)conditional tasks, a fair comparison is, in principle, possible only if the potential confound of a difference in number of underlying rules or S-R mappings is eliminated. However, such a confound does not seem to play a major role in tasks in which the participants have reached a relatively high level of performance accuracy.

\section{AUTHOR NOTE}

We thank Gosia Konopko, Jeroen Roor, Lorena Giménez, and Anna Allepuz for collecting the data reported in this article. Address correspondence to J.H.R. Maes, NICI/Biological Psychology, Radboud University Nijmegen, P.O. Box 9104, 6500 HE Nijmegen, The Netherlands (e-mail: r.maes@nici.ru.nl).

\section{REFERENCES}

Boettinger, C. A., \& D'Esposito, M. (2005). Frontal networks for learning and executing arbitrary stimulus-response associations. Journal of Neuroscience, 25, 2723-2732.

BoURne, L. E., JR. (1967). Learning and utilization of conceptual rules. In B. Kleinmuntz (Ed.), Concepts and the structure of memory (pp. 1-32). New York: Wiley.

Feldman, J. (2000). Minimization of Boolean complexity in human concept learning. Nature, 407, 630-633.

FELdman, J. (2003). The simplicity principle in human concept learning. Current Directions in Psychological Science, 12, 227-232.

FELdman, J. (2006). An algebra of human concept learning. Journal of Mathematical Psychology, 50, 339-368.

Halford, G., Wilson, W. H., \& Phillips, S. (1998). Processing capacity defined by relational complexity: Implications for comparative, developmental, and cognitive psychology. Behavioral \& Brain Sciences, 21, 803-864. 
Haygood, R. C., \& Bourne, L. E., JR. (1965). Attribute- and rulelearning aspects of conceptual behavior. Psychological Review, 72, 175-195.

Maes, J. H. R., \& Vossen, J. M. H. (2001). Acquisition of discriminations involving ambiguous or non-ambiguous features: An evaluation of two configural learning models. Behavioural Processes, 53, 191-201.

PeARCE, J. M. (1987). A model for stimulus generalization in Pavlovian conditioning. Psychological Review, 94, 61-73.

PeARCE, J. M. (1994). Similarity and discrimination: A selective review and a connectionist model. Psychological Review, 101, 587-607.

RESCORLA, R. A. (1973). Evidence for "unique stimulus" account of configural conditioning. Journal of Comparative \& Physiological Psychology, 1985, 331-338.

Rescorla, R. A., \& Wagner, A. R. (1972). A theory of Pavlovian conditioning: Variations in the effectiveness of reinforcement and nonreinforcement. In A. H. Black \& W. F. Prokasy (Eds.), Classical conditioning II: Current research and theory (pp. 64-99). New York: Appleton-Century-Crofts.

Swartzentruber, D. (1995). Modulatory mechanisms in Pavlovian conditioning. Animal Leaning \& Behavior, 23, 123-143.

(Manuscript received June 27, 2006; revision accepted for publication June 21, 2007.) 